\title{
Five-Membered Nitrogen Heterocyclic Compounds
}

\author{
Ahmad Sazali Hamzah, ${ }^{1}$ Zurina Shaameri, ${ }^{1}$ and Suleyman Goksu ${ }^{2}$ \\ ${ }^{1}$ Institute of Science, Universiti Teknologi MARA, 40450 Shah Alam, Selangor, Malaysia \\ ${ }^{2}$ Chemistry Department, University of Attartuk, 25400 Erzurum, Turkey \\ Correspondence should be addressed to Ahmad Sazali Hamzah; asazali@salam.uitm.edu.my
}

Received 21 November 2013; Accepted 21 November 2013

Copyright (c) 2013 Ahmad Sazali Hamzah et al. This is an open access article distributed under the Creative Commons Attribution License, which permits unrestricted use, distribution, and reproduction in any medium, provided the original work is properly cited.

Five-membered nitrogen heterocycles with a pyrrolidine moiety or pyrrolidine derivatives are often found in many bioactive molecules. Pyrrolidine, which is also known as tetrahydropyrrole, is a cyclic secondary amine with a fivemembered heterocycle containing carbon atoms and nitrogen. Pyrrolidine can be prepared from 1,4-diaminobutanes by the action of acids. Furthermore, it can act as a strong base, and it is often used in homogeneous nonaqueous reactions.

Major fractions of organic compounds isolated from nature are comprised of nitrogen heterocycles. Such fivemembered nitrogen heterocycles with a pyrrolidine ring system include nicotine, tryptamine, and vinblastine and possess considerable biochemical, pharmaceutical, and agricultural importance. These natural compounds may have hydroxyproline, 2-pyrrolidone, streptopyrrolidine, or diphenylprolinol rings as part of their well-defined conformations.

Apart from having significant structural features in many naturally occurring bioactive natural products, these ring systems can act as versatile intermediates towards the synthesis of more complex medicinally important compounds such as aniracetam, doxapram, cotinine, clausenamide, lactacystin, detoxine, and codonopsinine. These compounds have received much attention lately due to their diverse medicinal properties such as antibacterial, antibiotics, antitumor, and cytotoxic effects.

Constructing highly functionalized heterocyclic compounds would seem to be essential and significant prior to furnishing many of these biologically active natural constituents. Preparing enantiopure polysubstituted pyrrolidine derivatives can even be a more challenging task. A number of stereoselective methods for the synthesis of polysubstituted pyrrolidines have been reported in the last decade. This is due to the fact that the demand for enantiomerically pure drugs, agrochemicals, and food additives is rising, because pure enantiomers are often more target specific and have fewer side effects than racemic mixtures. Furthermore, the global market for chiral drugs alone currently stands at USD 100 billion and steadily grows at the rate of $9 \%$ per annum.

During the synthesis of a complex product consisting of several steps, it is essential for practical and economical reasons to introduce the proper stereochemistry in an early stage of the synthesis. This can be achieved by utilizing chiral building blocks that are enantiomerically pure and have functionalities that allow them to be transformed in the desired products. Nevertheless, stereocontrolled synthetic strategies will also have to be employed to acquire the potential products.

Diverse synthetic knowledge and chemical possibilities or transformations would be inevitably explored when conducting research in such area. During such synthetic processes, novel pharmacological agents beneficial in curing human diseases as well as new scientific findings may well be generated.

Research accounts in the field of organic chemistry and synthesis of five-membered nitrogen heterocyclic compounds (both aromatic and nonaromatic), as well as natural products with such heterocyclic systems, submitted and accepted in this publication complement the required standards and features of the journal.

The articles generally contain definitive and comprehensive reports of significant findings obtained via original work in heterocyclic chemistry. Some display novel synthetic methodology towards active heterocyclic materials which include efficient route, reproducible methodology, stereospecific materials, high product yields, and enantiomeric excess. Some works had also reported on findings from biological 
studies. Scientifically and fundamentally sound manuscripts with sufficient scientific data and evidence are highly considered.

Ahmad Sazali Hamzah Zurina Shaameri Suleyman Goksu 

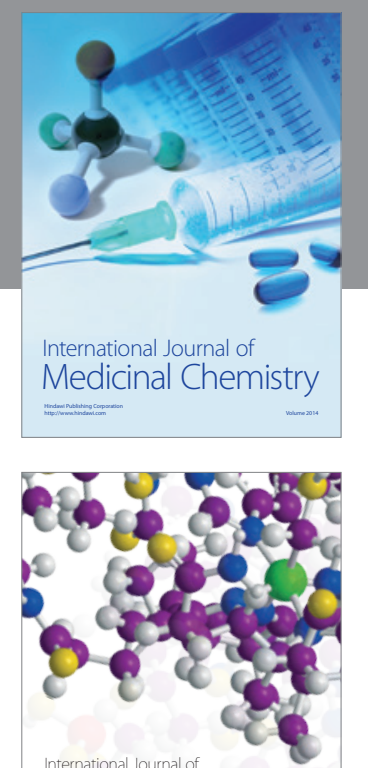

\section{Carbohydrate} Chemistry

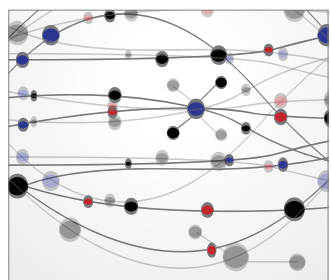

The Scientific World Journal
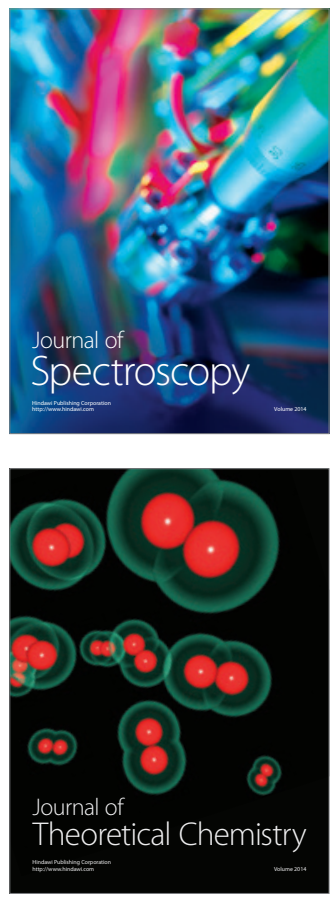
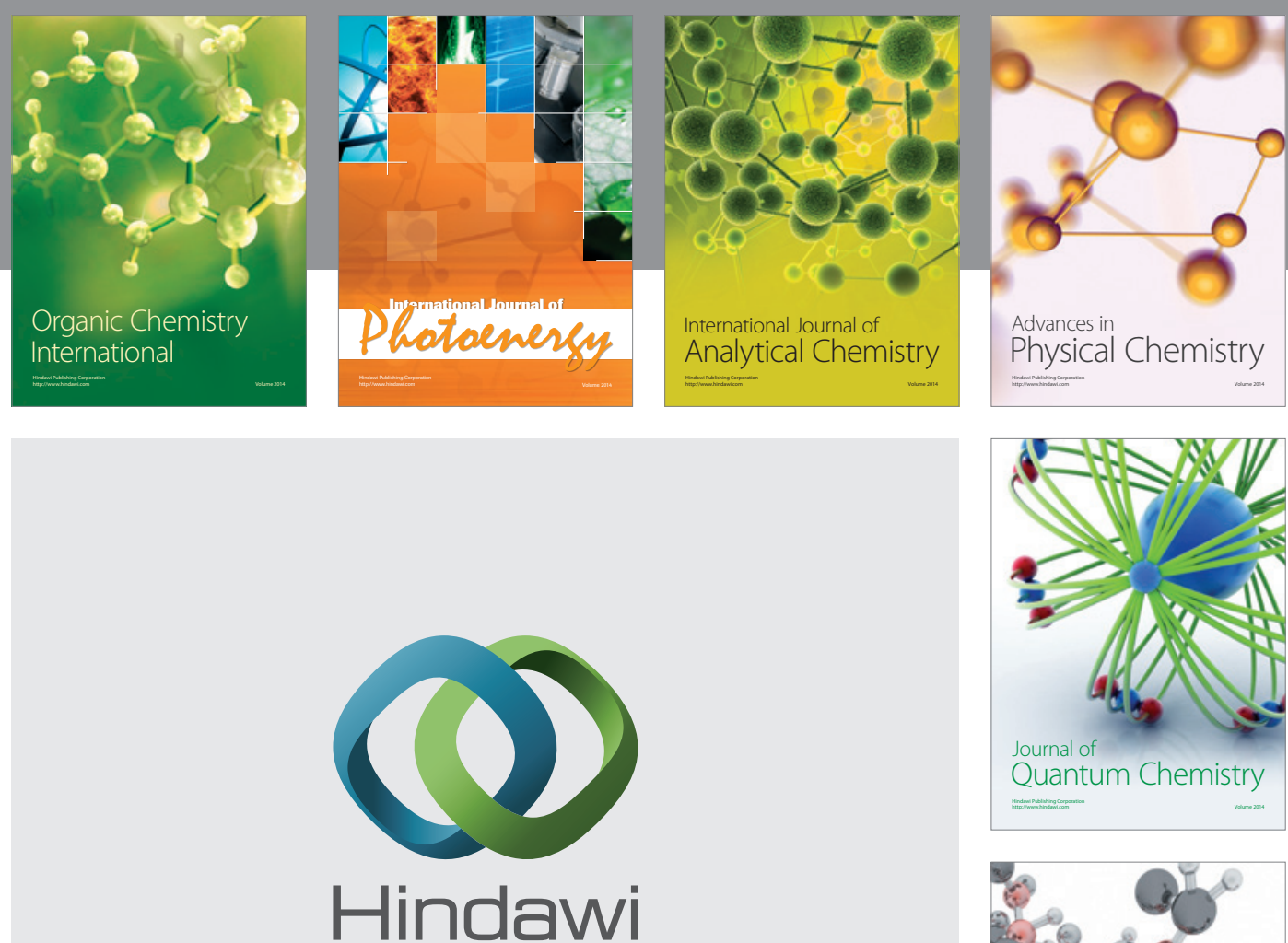

Submit your manuscripts at

http://www.hindawi.com

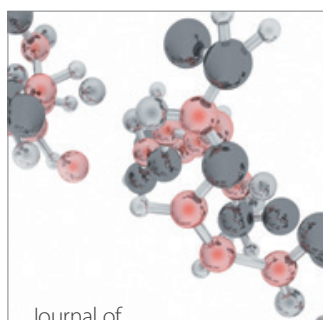

Analytical Methods

in Chemistry

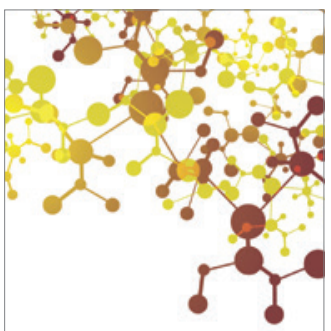

Journal of

Applied Chemistry

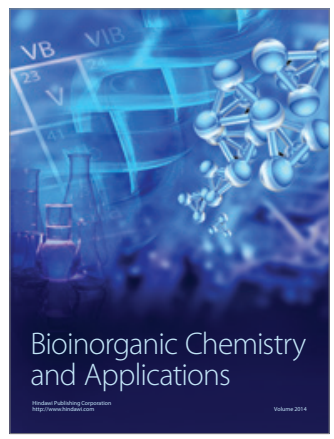

Inorganic Chemistry
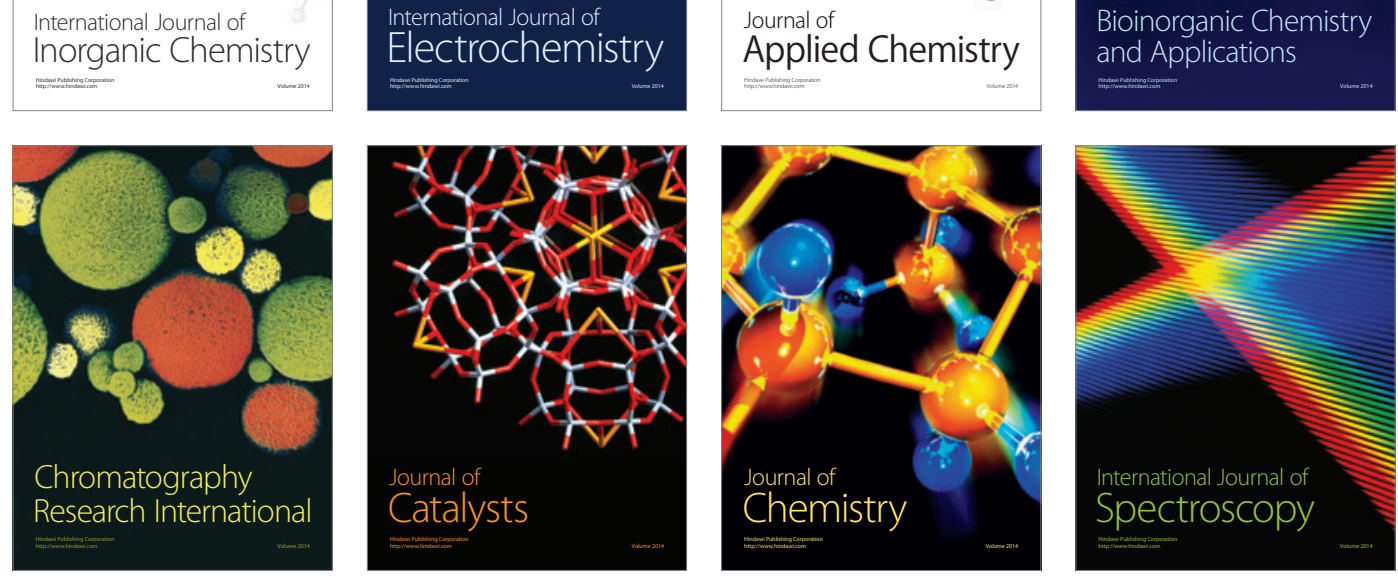